\title{
Spontaneous heterotopic pregnancy presenting with tubal rupture
}

\author{
Sukhwinder Kaur Bajwa ${ }^{1}$, Sukhminderjit Singh Bajwa ${ }^{1}$, Ram Nagar $^{1}$, Harkiran Kaur ${ }^{1}$ \\ Sri Lanka Journal of Obstetrics and Gynaecology 2010; 32: 41-43
}

\begin{abstract}
Heterotopic gestation, although common with assisted reproductive techniques, is very rare in natural conception. There are many variables in heterotopic pregnancy which makes the diagnosis of ectopic pregnancy a very challenging task. Nearly $85 \%$ of these cases go undiagnosed until there is rupture of the ectopic pregnancy that accompanies the intrauterine pregnancy. Though the presence of an intrauterine gestation reliably excludes the possibility of an ectopic gestation, it does not rule out a heterotopic pregnancy. So a rigorous sonographic examination of the adnexae and cul de sac is required even in the presence of an intrauterine gestation. Every clinician, who is treating women in reproductive age group, should keep this clinical catastrophe in mind for accurate and early diagnosis so as to do timely intervention which can decrease maternal morbidity and mortality. We present a rare case of heterotropic pregnancy after natural conception that was misdiagnosed to be a twin pregnancy until she presented with haemoperitoneum from ruptured tubal pregnancy.
\end{abstract}

Key words: Heterotopic pregnancy, ectopic gestation, tubal rupture.

\section{Introduction}

A coexistence of an extra- and intrauterine pregnancy is defined as heterotopic pregnancy. It has been traditionally regarded as a very rare event with an estimated incidence of 1 in 30,000 pregnancies $^{1}$. It rarely occurs in spontaneous conception cycles but recently, especially with the advent of assisted reproductive procedures, the rate has jumped to an alarming extent as high as 1 in 100. Heterotopic pregnancies are a diagnostic and a therapeutic challenge for obstetricians. If the pregnancy continues without diagnosis, a life-threatening situation can

\footnotetext{
${ }^{1}$ Adesh Institute of Medical Sciences and Research Bathinda, Punjab, India.

Correspondence: Sukhwinder Kaur Bajwa

E-mail: sukhwinderbajwa2001@gmail.com
}

occur. We present a case of spontaneous heterotopic pregnancy which presented with tubal rupture.

\section{Case report}

A 25-year old woman presented with pain and tenderness in right lower abdomen for two days for which she was taken to a nearby local hospital. She was diagnosed as a case of ruptured ectopic and was referred to our institute for further management. She had medical termination of pregnancy twenty days back at 8 weeks of amenorrhoea in a private hospital for twin pregnancy diagnosed on ultrasonography. There was no history of contraception or ovulation induction. She had one male child, 3 years old, who was delivered by caesarean section due to foetal distress.

On admission she was pale with a pulse rate of 100 per minute and blood pressure of 100/60 $\mathrm{mmHg}$. Laboratory findings revealed haemoglobin of $8.5 \mathrm{~g} \%$ and urine for pregnancy test was positive. Ultrasonography demonstrated right adnexal mass with internal cystic zone suggestive of 7 weeks and 5 days foetal pole with no cardiac activity. Uterine cavity showed debris with free fluid in pouch of Douglas. (Figure 1). The patient was immediately taken up for laparotomy. There was ruptured right sided tubal (ampullar) pregnancy with hemoperitoneum. Left fallopian tube was normal. Right sided salpingectomy was done with peritoneal lavage. Approximately $500 \mathrm{ml}$ of haemorrhagic fluid was collected. She was given two units of blood. Her recovery was uneventful and she was discharged in satisfactory condition.

\section{Discussion}

Heterotopic pregnancy is a form of multiple pregnancy with one embryo viably implanted in the uterus and the other being implanted elsewhere as an ectopic pregnancy. The incidence was originally estimated on theoretical basis to be 1 in 30,000 pregnancies. However, recent data indicates that the rate is much higher due to increased use of assisted reproduction techniques. It is estimated to be approximately 1 in 7000 overall and as high as 1 in 900 with ovulation induction ${ }^{1,2}$. The increased incidence of multiple pregnancies with ovulation induction and in-vitro fertilization increases the risk of both ectopic and heterotopic gestation. The hydrostatic forces generated during embryo transfer 

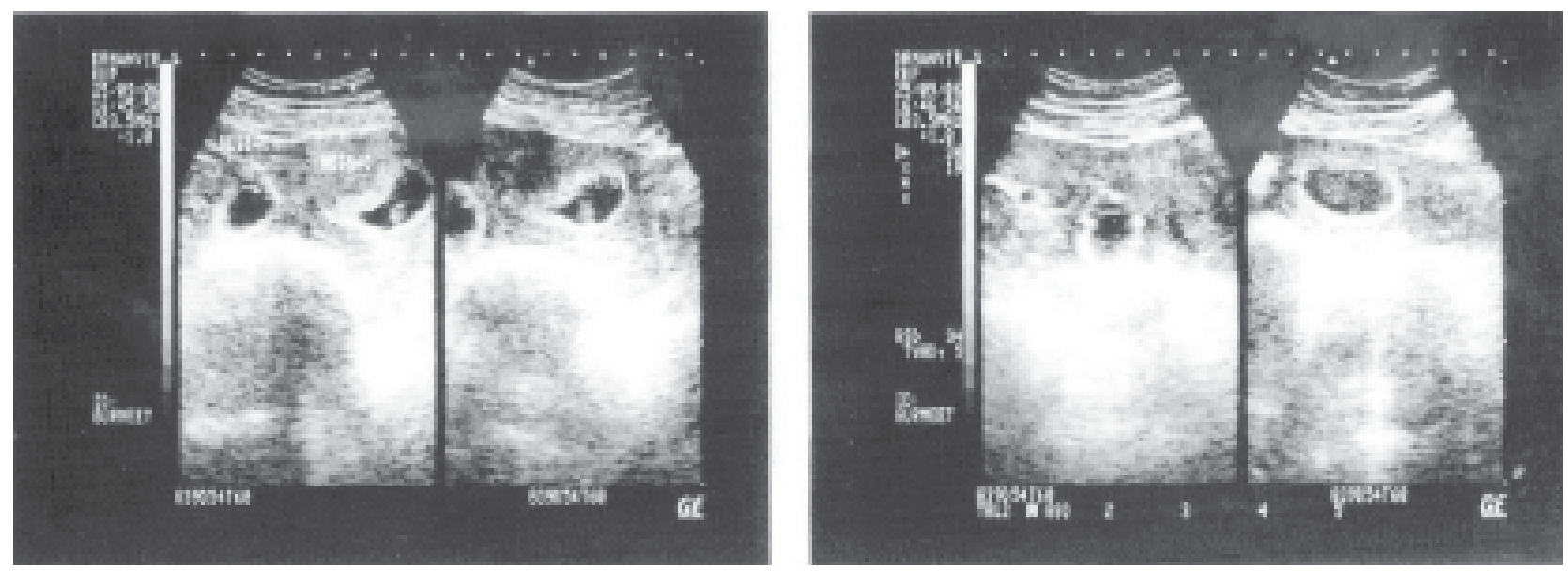

Figure 1. Ultrasonographic appearance of a heterotopic gestation.

may also contribute to the increased risk ${ }^{1}$. Risk factors which increase the frequency of heterotopic pregnancy are pelvic inflammatory disease, intrauterine devices, assisted reproduction techniques, endometriosis, sexually transmitted diseases and tubal surgeries. Indeed, any of the factors predisposing a patient to an increased risk of ectopic pregnancy and/or multiple gestations may contribute to heterotopic pregnancy ${ }^{3,4}$.

Heterotopic pregnancy can have various presentations. It should be considered more likely (a) after assisted reproduction techniques, (b) with persistent or rising chorionic gonadotropin levels after dilatation and curettage for an induced/spontaneous abortion, (c) when the uterine fundus is larger than for menstrual dates, (d) when more than one corpus luteum is present in a natural conception, and (e) when vaginal bleeding is absent in the presence of signs and symptoms of ectopic gestation ${ }^{5}$. A heterotopic gestation can also present as haematometra and lower quadrant pain in early pregnancy ${ }^{6}$. Most commonly, the location of ectopic gestation in a heterotropic pregnancy is the fallopian tube. However, cervical and ovarian heterotropic pregnancies have also been reported ${ }^{7}$.

Early diagnosis of heterotopic pregnancy is difficult because of subtle clinical symptoms. The most important method in diagnosing heterotopic pregnancy is high resolution transvaginal sonography. But most ultrasonographic reports do not encourage the search for coexistent ectopic pregnancy when evaluating intrauterine gestation because a heterotopic pregnancy is still thought to be extremely rare. For this reason majority of ectopic pregnancies are diagnosed by excluding an intrauterine pregnancy ${ }^{8}$. Whenever confusion arises regarding the intra- or an extrauterine pregnancy it is always wise to approach for transvaginal sonography which has a specificity rate of $73.7 \%$ and positive predictive value of $89.8 \%^{9}$. Transabdominal ultrasound is reported to be more useful than endovaginal ultrasound as it can visualize those areas which cannot be accessed by the latter. In fact, both methods are complementary ${ }^{10}$. High resolution transvaginal ultrasound with colour Doppler will be very helpful as the trophoblastic tissue in the adnexae in a case of heterotopic pregnancy shows increased flow with significantly reduced resistance index ${ }^{2}$. It is suggested that serum $\mathrm{B}-\mathrm{hCG}$ and progesterone levels can be useful besides ultrasonography, but excessive production of $\mathrm{B}-\mathrm{hCG}$ by placenta in intrauterine pregnancy can mask subnormal $\mathrm{s}-\mathrm{hCG}$ production as in ectopic pregnancy. Therefore, serial $\mathrm{B}-\mathrm{hCG}$ estimation is not useful in heterotopic pregnancy ${ }^{11}$.

The management of heterotopic pregnancy remains controversial. Surgical therapy has been the traditional mainstay but involves surgical and anaesthetic risks to both the mother and intrauterine pregnancy if it is to be continued ${ }^{12}$. Laparoscopic management is preferable over laparotomy in patients with a suspected ectopic pregnancy along with a documented intrauterine pregnancy because it involves minimal manipulation of the uterus ${ }^{4}$. When patients are haemodynamically unstable or come with shock, laparotomy is the treatment of choice. A conservative approach includes ultrasound guided local infiltration of methotrexate, mifepristone, prostaglandins, $\mathrm{KCl}$ and also aspiration of ectopic gestation via transvaginal route. This method can be used safely and effectively to manage patients who are clinically stable and where a heterotopic pregnancy is recognized relatively early in gestation $^{12,13}$. But generally methotrexate, mifepristone and prostaglandins are not sound options in the treatment of ongoing heterotopic pregnancy as these drugs are teratogenic ${ }^{14}$. In heterotopic pregnancy, maternal mortality is $1 \%$ and intrauterine foetal mortality rate is $45-65 \%{ }^{15}$. So early diagnosis of heterotopic pregnancy has a great importance as far 
as patient's mortality, morbidity, future fertility and continuation of a healthy intrauterine pregnancy is concerned.

\section{References}

1. Lyons EA, Levi CS, Sidney M. Dashefsky in Diagnostic Ultrasound. In: Rumak CM, Wilson SR, Charboneau WK, editors. 2nd ed. Volume 2, Mosby: 1998. p. 999.

2. Glassner MJ, Aron E, Eskin BA. Ovulation induction with clomiphene and the rise in heterotropic pregnancies: A report of two cases. J Reprod Med 1990; 35: 175-8.

3. Mistry BM, Balasubramaniam S, Silverman R, Sakabu SA, Troop BR. Heterotopic pregnancy presenting as an acute abdomen: A diagnostic masquerade. Am Surg 2000; 66(3): 307-308.

4. Perkins JD, Mitchell MR. Heterotopic pregnancy in a large inner-city hospital: A report of two cases. J Natl Med Assoc 2004, 96: 363-6.

5. Multifetal Ectopic Pregnancy, in Chapter 34. Ectopic Pregnancy, text book of - Williams Obstetrics, 21st ed. p.888-9.

6. Cheng PJ, Chueh HY, Qiu JT. Heterotopic pregnancy in a natural conception cycle presenting as hematometra. Obstet Gynecol 2004; 104: 195-8.

7. Hirose M, Nomura T, Wakuda K, Ishguro T, Yoshida Y. Combined intrauterine and ovary pregnancy: A case report. Asia Ocaena J Obstet Gynaecol 1994; 20: 25.
8. Peleg D, Bar-Hava I, Neaman-Leavin M, Ashkena, BenRafaelz IJ. Early diagnosis and successful non surgical treatment of viable combined intrauterine and cervical pregnancy. Fertil Steril 1994; 62: 405.

9. Mishra A, Youssefzadeh D, Parente JT. Heterotopic pregnancy. Female Patient 1998, 23: 39-42.

10. Chama CM, Obed JY, Ekanem IA. Transvaginal ultrasounds scan versus laparoscopy in the diagnosis of suspected ectopic pregnancy. J of Obstet and Gynecol 2001; 21(2): 184-6.

11. Qusehal A, Mamouchi H, Ghazli M, Kadiri R. Heterotopic pregnancy: Value of transabdominal sonography. J Radiol 2001; 82: 851-3.

12. Scheiber MD, Cedars MI. Successful non-surgical management of a heterotropic an abdominal pregnancy following embryo transfer with cryopreserved-thawed embryos. Hum Reprod 1999, 14: 1375-7.

13. Oyawoyea S, Chanderb B, Pavlovicb B, Hunterb J, Gadirb AA. Heterotropic pregnancy: Successful management with aspiration of cornual/interstitial gestational sac and instillation of small dose of methotrexate. Foetal Diagnosis and Therapy 2003; 18(1).

14. Scheiber MD, Marcelle I. Cedars case report: Successful non-surgical management of a heterotropic abdominal pregnancy following embryo transfer with cryopreservedthawed embryos. Human Reproduction 1999; 14: 1375-7.

15. Schenker J, Ezra Y. Complications of assisted reproductive techniques. Fertil Steril 1994; 61: 411-22. 